\title{
Die Einteilung der acidimetrischen und alkalimetrischen Indikatoren.
}

\author{
Von \\ JULIUS WAGNER.
}

Vor etwa 2 Jahren hat F. GLaser der ,freien Vereinigung bayrischer Vertreter der angewandten Chemie" ein Referat erstattet über die Indikatoren der Acidimetrie und Alkalimetrie, ${ }^{1}$ in der er eine Einteilung dieser Indikatoren gegeben hat. Diese liegt nunmehr in ausführlicherer Form vor. ${ }^{2}$ So aufserordentlich dankenswert dieser Versuch auch ist, so kann doch bezweifelt werden, ob er als gelungen zu betrachten ist. Von einer glücklich gewählten Einteilung lassen sich verschiedene Vorteile erwarten. Einmal die bessere Übersicht in die Wirkungsweise und die Verwendbarkeit des Indikators, dann aber die Möglichkeit, für die Praxis neue Indikatoren nach Analogie der bereits vorhandenen systematisch aufzusuchen und neu herzustellen. Sollen diese Vorteile erreicht werden, so mul's in erster Linie der chemische Charakter des Indikators als Einteilungsgrund genommen werden, aus diesen Eigenschaften ergiebt sich die praktische Brauchbarkeit ohne weiteres.

GLASER hat aber seine Einteilung nach dem praktischen Gesichtspunkte der Empfindlichkeit aufgestellt und komint so zu drei ziemlich willkürlich von einander unterschiedenen Gruppen, die, wie er selbst zugiebt, nicht scharf von einander getrennt sind.

Er unterscheidet als erste Gruppe die Indikatoren von schwach basischer oder ausgeprägt saurer Natur. Erstere bilden nur mit starken Säuren beständige Indikatorsalze, die andere Farbe zeigen als der Indikator selbst, und schon durch schwache Basen zersetzt werden. Stark saure Indikatoren - sehr starke sind natürlich aus-

1 Zeitschr. analyt. Chem. 38, 273.

${ }^{2}$ Graser, Indikatoren der Acidimetrie u. Alkalimetrie. Wiesbaden 1901. 
geschlossen ${ }^{1}$ - bilden selbst mit schwachen Basen Salze, die nur durch starke Säuren gespalten werden. So sind die Indikatoren von GLASER's erster Gruppe empfindlich gegen Alkali, unempfindlich gegen Säuren. Die Gruppe enthält: Methylviolett, Jodeosin, Tropüolin 00, Methyl- und $\ddot{A}$ thylorange, Helianthin, Dimethylamidoazobenzol, Kongorot, Benzopurpurin, Cochenille, Lackmoid.

Die zweite Gruppe enthält Verbindungen von Säurecharakter, zumeist oder wohl ausschliefslich Phenole. Sie sind schwache Säuren, deshalb empfindlicher gegen Säuren, weniger empfindlich gegen Basen, d. h. ihre Salze werden auch schon durch schwächere Säuren zerlegt, bilden sich aber nur durch stärkere Basen. Es gehören hierher Fluorescein, Phenacetolin, Alizarinsulfosaures Natrium, Hämatoxylin, Galleun, Alizarin, Orseille, Lackmus, p-Nitrophenol. Endlich die Indikatoren der dritten Gruppe sind noch schwächere Säuren, also sind ihre Salze noch leichter, mithin auch durch sehr schwache Säuren zerlegbar, sie bilden sich nur durch die stärksten Basen. Die Gruppe umfalst Rosolsäure, Tropäolin 000, Curcuma, Curcumin W, Flavescin, Phenolphtaleïn, $\alpha$-Naphtolbenzern und Poirriersblau $\mathrm{C}_{4} \mathrm{~B}$.

Diese Einteilung gewährt zweifellos einen gewissen praktischen Nutzen. Man wird, um mit starken Säuren zu titrieren Indikatoren aller drei Gruppen wählen können; je schwächer die Säure ist, mit der man titriert, einen umso säureempfindlicheren Indikator muls man verwenden, also einen der dritten Gruppe sich annähernden oder angehörenden.

Ähnlich liegen die Verhältnisse beim Titrieren mit Basen; je schwächer die Base ist, mit der man titriert, umso stärker mufs der Indikator gewählt werden. Die Natur der Base oder Säure, die man titriert, kommt bei der Wahl des Indikators nur mittelbar zur Geltung, insofern sie die Wahl der Säure oder Base, mit der man titriert, beschränken. Denn je schwächer die Base oder Säure, desto stärker mufs die Säure oder Base sein, mit der die Neutralisation vollzogen wird, da sonst die Hydrolyse störend auftritt.

Bei dieser Einteilung ist aber, wie gesagt, lediglich die Empfindlichkeit berücksichtigt, in keiner Weise der chemische Charakter; die erste Gruppe enthält z. B. Säuren und Basen. Der chemische Charakter läfst aber verschiedene Eigentümlichkeiten der Indikatoren leicht verständlich werden.

${ }^{1}$ Siehe die Begründung der Theorie der Indikatoren bei Ostwar.D, Wissenschaftl. Grundlagen. Leipzig 1897, S. 116, bezw. 1901. 
Als erster Einteilungsgrund der Indikatoren scheint mir die Art der Ionenbildung verwendet werden zu müssen, dem sich innerhalb der einzelnen Gruppen die Empfindlichkeit, soweit sie auf der Quantität der Ionenbildung beruht, ${ }^{1}$ erst als zweiter Einheitsgrund anschliefst.

Die Qualität der Ionenspaltung gestattet auch wieder zwei Einteilungen. Wir haben zu unterscheiden zwischen Auionen und Kationen und zwischen einwertigen und mehrwertigen Ionen. Letztere Unterscheidung scheint mir als erster Einteilungsgrund am zweckmäfsigsten.

Ich unterscheide also:

A) Indikatoren mit einwertigem charakteristischen Ion.

1. mit einwertigem charakteristischen Anion,

2. mit einwertigem charakteristischen Kation.

B) Indikatoren mit mehrwertigem Ion.

1. mit positivem und negativem Ion (amphotere Elektrolyte). ${ }^{2}$

2. mit ein- und zweiwertigem Anion oder Kation.

In praktischer Beziehung unterscheiden sich die Hauptgruppen, wie ich vorläufig betonen will, hauptsächlich durch das Fehlen bezw. das Auftreten einer Zwischenfarbe, die unter Umständen die Bestimmung verschiedener Anionen bezw. Kationen mit Hülfe desselben Indikators gestattet.

Gerade diese praktisch bedeutsamen Körper kommen in der GLaSER'schen Einteilung gar nicht zur Geltung.

Betrachten wir nun die einzelnen Gruppen meiner Einteilung, so kommt für die Hauptgruppe A zunächst das von Glaser nach OstwaLD's Vorgang Ausgeführte zur Geltung.

1. Indikatoren mit einwertigem charakteristischem Anion.

In sauren Lösungen ist der Indikator wenig dissociiert, umsoweniger, je schwächer er selbst als Säure ist. In dem Malse als die Wasserstoffionen verschwinden, wächst seine Dissoziation und zwar umso langsamer je schwächer seine Säurenatur ist, im idealen Grenzfalle, wie er beim Phenolphtaleyn etwa erreicht ist, erst, wenn durch einen Überschufs von Hydroxylion eine praktisch erkennbare Dissoziation erreicht wird. Der Indikator wird also umso empfind-

${ }^{2}$ Die Empfindlichkeit hängt aufserdem noch von dem Farbenkontrast der in Betracht kommenden Moleküle bezw. Ionen ab.

2 Bredia, Zeitschr. Elektrochem. 6, 33. 
licher, je schwächer er als Säure ist. Da aber bei schwachen Säuren die Hydrolyse hohe Grade erreicht, d. h. ein verhältnismälsig grolser Überschufs von Hydroxylionen notwendig ist, um das charakteristische Anion auftreten zu lassen, so sind starke Basen zur Titration zu verwenden; mit schwachen Basen kann erst mit verbältnismäfsig starken Indikatorsäuren titriert werden, z. B. Ammon mit p-Nitrophenol.

Ganz gleiche Verhältnisse finden wir, aber in umgekehrter Richtung, wenn wir eine Base mit einem Indikator der ersten Gruppe durch eine Säure messen wollen.

Hier findet sich in der Lösung das charakteristische Ion zunächst vor, auf Zusatz einer Säure folgt im wesentlichen Wasserbildung, aus ihren Wasserstoffionen und den Hydroxylionen der Basis. Erst wenn letztere sich mindern, können die zutretenden Wasserstoffione mit dem charakteristischen Anion zur nichtdissoziierten Säure zusammentreten und den Farbenwechsel hervorrufen. Dies wird umso früher, im idealen Grenzfalle schon durch den geringsten Überschufs von Wasserstoffionen erfolgen, je schwächer die Indikatorsäure ist. Handelt es sich um eine starke Indikatorsäure, so bringt erst ein grölserer Überschuls an Säure den Farbenwechsel hervor. Grundsätzlich sind also die schwachen Indikatorsäuren vorzuziehen, soweit dies die Rücksicht auf die notwendige Stärke des Farbenunterschiedes zulälst.

Die Hydrolyse mufs natürlich auch hier berücksichtigt werden. Starke Basen können deshalb mit starken und schwachen Säuren und beliebigem Indikator titriert werden, schwache Basen aber nur mit starken Säuren.

Die Verwendung der stärksten Indikatorsäuren ist, weil die Bildung des charakteristischen Ions schon in ziemlich sauren Lösungen erfolgt, oder weil es erst durch einen Säureüberschufs verscbwindet, nur unter besonderen Vorsichtsmalsregeln möglich. So z. B. beim Jodeosin, wo durch Schütteln der Lösung mit Äther ihr das nichtdissoziierte Jodeosin entzogen wird, so dafs selbst in der noch eben sauren Lösung nur eine äufserst geringe Menge Jodeosin zurückbleibt, deren Farbe noch in Betracht kommt.

In diese Gruppe A 1 gehören von dem bei Glaser genannten Indikatoren: ${ }^{1}$ Jodeosin, Cochenille, Fluorescin, Alizarin, Orseille, p-Nitrophenol, Rosolsäure, Tropäolin 000, Curcuma, Curcumin W.

Graser, Indikatoren, S. 5. 
Flavescin, Lackmoid, Lackmus, Phenolphtalein und Poirriersblau, also weitaus die meisten Indikatoren. Die Reihenfolge entspricht der Stärke als Säure. Jedoch ist zu bemerken, dafs diese von GLASER aufgestellte Folge zumeist auf Schätzung beruht und wirkliche Messungen nur für wenige der genannten Stoffe vorliegen, und es kann deshalb die Anwendung gegenüber anderen Säuren oder gegenüber Basen jeweils nur durch den Versuch entschieden werden. Wünschenswert wäre die Ausarbeitung einer Tabelle, aus der sich die Brauchbarkeit der Indikatoren gegenüber Basen bezw. Säuren von bestimmter und bekannter Stärke ergäbe.

2. Indikatoren mit einwertigem Kation.

Für diese Indikatoren gilt mit den notwendigen Änderungen alles vorher Gesagte.

Erwünscht sind auch hier schwache Elektrolyte, es kommen aber auch nur solche in Betracht. Denn diese Untergruppe enthält nur zwei bekanntere Indikatoren, das Methylviolett und das Dimethylamidoazobenzol, die Muttersubstanz des Methylorange. Diese Verbindung färbt an sich gelb, saure Lösungen sind rot.

In die zweite Hauptgruppe B gehören die Indikatoren mit mehrwertigen Ionen.

1. Indikatoren mit verschiedenen charakteristischen Ionen. Die Indikatoren zeigen die Eigentümlichkeiten, dafs sie in sauren und in alkalischen Lösungen verschieden gefärbte Ionen bilden, in ersteren Kationen und in letzteren Anionen, sie verhalten sich also etwa wie das Aluminiumhydroxyd oder Zinkhydroxyd. Es ergiebt sich hieraus die Möglichkeit einer Zwischenfarbe, ${ }^{1}$ die dem neutralen Körper angehört. Thatsächlich ist eine solche $Z$ wischenfarbe bei einzelnen Gliedern dieser Gruppe zu beobachten, so z. B. bei dem bekanntesten der hierher gehörigen Indikatoren, dem Methylorange. In der sauren Lösung findet es sich als rotes Kation ${ }^{\mathrm{NH}}\left(\mathrm{CH}_{3}\right)_{2} \cdot \mathrm{C}_{6} \mathrm{H}_{4} \cdot \mathrm{N}_{2} \cdot \mathrm{C}_{6} \mathrm{H}_{4} \mathrm{SO}_{3} \mathrm{H}$, also eines. Ions vom Ammoniumtypus, in der alkalischen Lösung als gelbes Anion $\mathrm{N}\left(\mathrm{CH}_{3}\right)_{2} \cdot \mathrm{C}_{6} \mathrm{H}_{4} \cdot \mathrm{N}_{2} \cdot \mathrm{C}_{6} \mathrm{H}_{4} \cdot \mathrm{SO}_{3}^{\prime}$, also eines Ions vom Ammoniaktypus. Dies ist jetzt wohl allseitig anerkannt. ${ }^{2}$ Zwischen den beiden Farben macht sich eine Zwischenfarbe geltend, wenn schwache Basen oder Säuren zugegen sind. Diese Zwischenfarbe mufs in irgend einer Weise durch den Neutralkörper hervorgerufen werden, gleichgültig ist, wie man sie aus dem Verhalten des Neutralkörpers er-

1 Nicht Übergangsfarbe, wie sie bei unscharfem Umschlag infolge geringer Emptindlichkeit in Gruppe A auftreten kann.

2 Bredig und Winkelblech, Zeitschr. Eleltrochem. 6, 35 
klärt. Ich will aber beiläufig auf diesen besonders von KÜsTER ${ }^{1}$ behandelten Punkt eingehen. Nach KÜsTER kann die Übergangsfarbe nicht dem undissoziierten Farbstoff - also dem Neutralkörper - zugeschrieben werden, da es wenig wahrscheinlich ist, dals bei der grolsen Verdünnung, in der sich der Farbstoff bei der Titration befindet und bei der verhältnismäfsigen Stärke der Säure, solcher vorfindet. Er macht deshalb die Annahme eines Zwitterions 'H.N $\left(\mathrm{CH}_{3}\right)_{2} \cdot \mathrm{C}_{6} \mathrm{H}_{4} \cdot \mathrm{N}_{2} \cdot \mathrm{C}_{6} \mathrm{H}_{4} \cdot \mathrm{SO}_{3}^{\prime}$, das er aus dem Farbstoff' selbst entstehen läfst, indem sich die von der Sulfogruppe abdissoziierenden Wasserstoffionen an das Stickstoffatom unter Bildung des Ammoniumions anlagern. Die Farbe des Zwitterions soll schwach rot sein und erst hervortreten, wenn durch überschüssige Wasserstoffionen alles Ion $\mathrm{N}\left(\mathrm{CH}_{3}\right)_{2} \cdot \mathrm{C}_{6} \mathrm{H}_{4} \cdot \mathrm{N}_{2} \cdot \mathrm{C}_{6} \mathrm{H}_{4} \cdot \mathrm{SO}_{3}^{\prime}$ in das $\mathrm{Z}$ witterion übergeführt ist. Ist Kohlensäure in erheblicher Menge zugegen, so bekommt man thatsächlich eine Mischfarbe zwischen gelb und rot, die KüsteR durch die allmähliche Entwickelung der Wasserstoffionen der Kohlensäure erklärt, die sich in dem Malse entwickeln, als Karbonat bezw. Bikarbonat zersetzt wird. Er scblägt deshalb vor, nicht das entschiedene Rot als Endpunkt zu betrachten, sondern die Mischfarbe, die eine Methylorangelösung beim Sättigen mit Kohlensäure annimmt. Praktisch ist hiergegen nichts einzuwenden, wohl aber gegen die Deutung des zu Grunde liegenden Versuchs. Es scheint mir nämlich übersehen, dais nicht das Methylorange eigentlich der Indikator ist, sondern die Säure, die dem Farbstoff zu Grunde liegt und dafs deren Eigenschaften berücksichtigt werden mülsten. Diese Säure selbst, die in Wasser sehr schwer löslich ist, hat eine deutliche rötliche Eigenfarbe, wie sie auch in den konzentrierteren Methylorangelösungen hervortritt. Wahrscheinlich ist hier ziemlich merkbare Hydrolyse vorhanden, denn erst beim vorsichtigen Zusatze von Kalilauge tritt reine Gelbfärbung ein, Zusatz weiterer Kalilauge fällt gelbes Salz. Leitet man deshalb in eine gelbe Lösung von Methylorange Kohlensäure bis zur Sättigung, so wird etwas der nichtdissoziierten freien Säure gebildet ${ }^{2}$ und deren Farbe mischt sich dem Gelb des Anions

${ }^{1}$ Küster, Z. anorg. Chem. 13, 135, Ann.

2 Bei dem Küsten'schen Versuch, Verfärbung einer durch einen Tropfen Methylorange gefärbten Wassermenge von $50 \mathrm{ccm}$ durch Sättigen mit Kohlensäure, haben wir das Mol Indikatorsäure in 300000 Litern, während die Kohlensäure 1/s normal ist. Bei Annahme von rund 1.5\% Dissoziation (nach WALEER, Zeitschr. phys. Chem. 32, 137 berechnet) ergiebt das eine 1/6000 normale Wasserstoffionenlösung, so dals eine Verfärbung schon eintreten kann, selbst wenn die Säure des Methylorange ziemlich stark ist. 
zur Übergangsfarbe bei, es kann aber die Kohlensäure das gelbe Salz nicht vollständig zersetzen und es bleibt bei der Mischfarbe. Stärkere Wasserstoffionenkonzentration lälst alles gelbe Anion verschwinden und führt dann in das rote Kation über. Zu letzterer Annahme wird man notwendig geführt durch das Verhalten des Dimethylamidoabenzols, bei dem die Rotfärbung durch Säuren nicht anders erklärt werden kann, wie schon GLASER bemerkt. Anscheinend hat die Farbstoffsäure selbst und das Kation einen ähnlichen Farbton, wegen der aufserordentlichen Schwerlöslichkeit lälst sich dies nicht genau prüfen. Für meine Auffassung der Wirkung des Methylorange scheinen mir nun die Verhältnifse bei einem ganz analog gebauten Farbstoff, dem Kongorot, zu sprechen. Hier haben wir noch mehr im einzelnen unterscheidbare Farbenverhältnisse.

Der Farbstoffstoff selbst ist wiederum ein Natriumsalz, die Lösung ist rotbraun gefärbt, Mineralsäure färbt blau und fällt im Überschufs einen blauen Farbstoff, der das salzsaure Salz der Säure des Kongorots darstellt, diese Säure selbst ist rotbraun und löst sich so in Wasser.

Bei der Titration findet ein Interschied zwischen der Titration mit Alkali oder Säure statt. In saurer Lösung verschwindet die blaue Farbe allmählich mit dem Zusatz des Alkali, durch Violett geht sie in Rot über. Letzteres ist die Farbe des neutralen Salzes, das Violett ist als Mischfarbe zwischen Blau und der Eigenfarbe der Säure zu betrachten. Diese kommt frühzeitig zum Vorschein, weil das Salz des Kongorotkations nur bei Überschufs von Säure besteht und sonst Hydrolyse eintritt. Dals dem so ist, folgt $u$. a. aus dem Verhalten des gefällten blauen Salzes beim Behandeln mit Wasser. ${ }^{1}$

Dementsprechend ist bei der Titration aus alkalischer Lösung der Übergang nicht scharf, so dafs es sich empfiehlt, überzutitrieren und mit Alkali zurückzugehen. Der Indikator wirkt also als Säure, für den Umschlag ist entscheidend der Übergang aus Violett bezw. Rotbraun in brennendes Rot, die basischen Eigenschaften des Indikators spielen nur eine nebensächliche Rolle. Ich komme also hier zu dem umgekehrten Schlusse wie GLasER, der beim Kongorot, um seine wenig rationelle Systematik zu retten, die Thatsachen sehr einseitig würdigt.

Wenn wir nun diese recht klaren Verhältnisse vom Kongorot auf das Methylorange übertragen, so können wir sagen: Die sauren

1 GLASER, Indikatoren, S. 56. 
Lösungen enthalten das rote Kation vom Ammoniumtypus; beim allmählichen Zusatz von Alkali tritt schliefslich Hydrolyse ein; neben dem roten Kation erscheint die gelbe Farbe des Anions vom Ammoniaktypus. Erst bei vollständigem Übergang tritt die rein gelbe Farbe auf. Der Umschlag in Gelb ist aber bei geringer Konzentration des Indikators und völlig kohlensäurefreien Lösungen scharf, weil die $Z$ wischenfarbe durch den Tropfen Normalalkali, der sie hervorruft, auch schon wieder verschwindet, ${ }^{1}$ und entspricht der vollständigen Überführung der vorhandenen Säure in ein Neutralsalz. Dagegen ist der Umschlag in Rot zwar scharf in kohlensäurefreien Lösungen, nicht aber in mit Kohlensäure gesättigten Lösungen. Diese scheidet die Farbstoffsäure wegen der Massenwirkung vorzeitig $a b$, ihre Farbe läfst dann die Mischfarbe entstehen, und wenn nur bis zu diesem Punkte titriert wird, sie wird - wie ich glaube die Titration zu früh abgebrochen, denn nach den Messungen von WINkELBLECH ${ }^{2}$ können wir der Kohlensäure wohl nicht die Bildung von roten Kationen zuschreiben.

Die von KÜsteR beobachtete Verfärbung der Methylorangelösung hat dieselbe Ursache, wie die gleiche Verfärbung beim Phenolphtalein, nur dafs sie hier entsprechend der geringeren Stärke dieses Indikators vollständig ist, so tritt, wie ich mich überzeugt habe, nicht nur bei dem Methylorange und Äthylorange, sondern auch bei den Indikatorsäuren selbst auf. Es tritt aber nur Verfärbung ein, das gelbe Anion verschwindet durch Kohlensäure nicht, wohl aber durch sehr geringe Mengen Salzsäure.

$\mathrm{Ob}$ übrigens die $\mathrm{Z}$ wischenfarbe, die man bei der Kongorotsäure nur gemischt mit dem Rot, beim Methylorange nur gemischt mit

${ }^{1}$ Bei Lösungen, die mit einem Tropfen Methylorange (1: 1000) gefärbt, haben wir zwischen Methylorange und der Hydroxyl- bezw. Wasserstoffionenmenge, die durch den umschlaggebenden Tropfen eingeführt wird, das Konzentrationsverhältnis $1: 300$ bei Normallösung, also $1: 30$ bei $1 / 10$ Normallösungen. Da die Wasserstoffionenkonzentration der gesättigten Kohlensäurelösung $1 / 6000$ normal ist (S. 143) und durch diese kein reines Rot erzengt wird, so folgt, dals die Wasserstoffionenkonzentration für den Umschlag grölser sein muls. In $50 \mathrm{ccm}$ kann also ein Tropfen 1/1 Säure, der eine Wasserstoffionenkonzentration von $1 / 1000$ normal entspricht, den Umschlag bewirken, nicht aber ein Tropfen $1 / 10$ Säure, der die Wasserstoffionenkonzentration nur auf $1 / 10000$ normal bringt. Beides entspricht der Erfahrung, verdünnt man genau eingestellte $1 / 1$ Lösungen auf $1 / 10$, so ändert sich der Wirkungswert um den Wert von ein bis zwei Tropfen.

${ }^{2}$ Nach mündlicher Mitteilung sind basische Eigenschaften beim Methylorange bezw. dessen Säure kaum mefsbar.

Z. anorg. Chem. XXVIr. 
dem Gelb sieht, als die Farbe der nichtdissoziierten Indikatorsäure betrachten mufs, bleibt zweifelhaft. Es ist ja möglich, dafs diese Säuren sich als Zwitterionen nach der KüstER'schen Annahme lösen, sie könnten auch, was ziemlich auf dasselbe herauskäme, als lactonartige Verbindung in Lösung gehen, also als Anhydrid der Dimethylammoniumazobenzolsulfonsäure. Hierzu bildet die Diazobenzolsulfosäure eine Analogie, und ich möchte glauben, dafs bei den kaum löslichen „Säuren" des Methyl- und Äthylorange der Fall so liegt. Die Säure des Kongorots ist leichter löslich und hat ausgesprochen saure Eigenschaften, wie schon die Möglichkeit, Ammon zu titrieren, beweist.

Für die Wirksamkeit der amphoteren Elektrolyte als Indikator kann folgendes gesagt werden. Für die Titration von sauer und alkalisch kommt die Säurequalität des Indikators in Betracht. Starke Säureindikatoren, wie Kongorot, Methyl- und Äthylorange, lassen sich auch bei der Titration mit schwachen Basen wie Ammoniak verwenden, schwache Säureindikatoren, versagen bei Ammoniak, doch sind solche nicht bekannt.

Die Schärfe des Umschlags hängt im übrigen wesentlich $a b$ von der Verschiedenheit der Farbe des reinen Indikatoranions von der des Säureions, wie sie neben dem nichtdissoziierten Säuremolekül erscheint.

Bei der Titration aus alkalischer Lösung zu sauer kommt die Stärke der basischen Eigenschaften in Betracht. Hinreichend starke Basen können einen starken Umschlag geben, bei weniger starken mischt sich die Farbe des basischen Ions mit der des Indikators selbst und der Umschlag leidet. Dies ist der Fall bei Kongorot, weniger bei Methylorange sowie anscheinend bei allen als Indikatoren vorgeschlagenen amphoteren Indikatoren.

Eine Zwischenfarbe tritt immer ein, wenn der Indikator eine Eigenfarbe hat; die Zwischenfarbe ergiebt sich aus der Mischung des basischen oder sauren Ions und der Eigenfarbe des Indikators, ob die Zwischenfarbe bemerkbar wird, hängt von dem Unterschied in der Stärke der beiden Indikatorionen ab.

Es sind deshalb amphotere Elektrolyte als Indikatoren ganz allgemein brauchbar für die Titration von sauer zu neutral; ob mit allen (Kongorot) oder nur mit starken Basen titriert werden kann, hängt ganz von der Stärke der Säureeigenschaften des Indikators ab. Das Kongorot entspricht allen Ansprüchen, die wir 
an einen Indikator für die Titration von sauer zu neutral stellen können, insbesondere wegen der verhältnismälsig grofsen Unempfindlichkeit gegen Kohlensäure; es lassen sich aber, weil die Indikatorsäure selbst ziemlich stark ist, nur relativ stärkere Säuren damit titrieren. Die Grenzen sind bis jetzt nicht festgestellt.

Für die Titration von alkalisch auf neutral können nur solche amphotere Elektrolyte ohne Störung durch die Mischfarbe verwendet werden, die noch so starke Basen sind, wie Methylorange.

In die Gruppe amphoterer Elektrolyte sind folgende Indikatoren zu rechnen: Tropäolin 00 (phenylamidoazobenzolsulfosaures Natrium), Methyl- und Äthylorange bezw. ihre Säuren (Helianthin), Kongorot, Benzopurpurin, also verhältnismäfsig wenige Stoffe. Die Reihenfolge hat keine Bedeutung, da naturgemärs diese Indikatoren in zwei Reihen geordnet werden müfsten; die eine für den Gebrauch zur Neutralisation alkalischer, die andere für den Gebrauch zur Neutralisation saurer Lösungen. Hierfür sind aber genügende Unterlagen nicht vorhanden.

Ich komme zur letzten Gruppe der Indikatoren, die von mir auf S. 140 als B 2 bezeichnet ist. Sie enthält die Stoffe, die verschiedene charakteristisch gefärbte Ionen gleicher Art, also nur Anionen oder Kationen liefert und bisher auf Säuren, also anionogene Stoffe, beschränkt ist.

Sie finden ihr anorganisches Analogon in Stoffen wie die Phosphorsäure, wo die Konzentration der Wasserstoffionen bei der Säure selbst und beim primären Salze in so weiten Grenzen verschieden ist, dals mit Hilfe verschiedener Indikatoren, Methylorange und Phenolphtaleïn, eine Bestimmung von Phosphorsäure neben Phosphat möglich ist. Bei den Indikatoren der Gruppe B, 2 sind nun die charakteristischen Ionen der Säuren verschieden gefärbt, so dals sie nacheinander auftreten. Zunächst bildet sich das eine, noch saure Ion, dann das zweite. Ihre Verwendung geschieht so, dals beim Titrieren aus saurer Lösung zunächst die Farbe des nichtdissoziierten Indikators vorliegt, die bei einer gewissen Wasserstoffionenkonzentration verschwindet, um der Farbe des ersten, noch sauren, charakteristischen Ions Platz $z \mathfrak{u}$ machen. Durch weiteren Alkalizusatz kann nun eine weitere anwesende - aber natürlich sehr viel schwächere - Säure bestimmt werden, die Beendigung der Titration giebt sich durch die Bildung des zweiten charakteristischen Indikatorions kund. Ein gutes Beispiel giebt die Alizarinsulfosäure, 
deren Natriumsalz als Indikator verwendet wird. In saurer, gelber Lösung ist die nichtdissoziierte Säure vorhanden, beim Neutralisieren erscheint zunächst Rotfärbung durch Bildung des Ions:

$$
\mathrm{C}_{6} \mathrm{H}_{4}<\underset{\mathrm{CO}}{\mathrm{CO}}>\mathrm{C}_{6} \mathrm{H} \cdot(\mathrm{OH})_{2} \cdot \mathrm{SO}_{3}^{\prime},
$$

durch mehr Alkali erscheint das violette Ion:

$$
\mathrm{C}_{6} \mathrm{H}_{4}<\underset{\mathrm{CO}}{\mathrm{CO}}>\mathrm{C}_{6} \mathrm{H}<\mathrm{O}_{\mathrm{O}_{2}^{\prime \prime}}^{\mathrm{SO}_{3}^{\prime}}
$$

oder wahrscheinlicher:

$$
\mathrm{C}_{6} \mathrm{H}_{4}<\underset{\mathrm{CO}}{\mathrm{CO}}>\mathrm{C}_{6} \mathrm{H}(\mathrm{OH})<\underset{\mathrm{O}^{\prime}}{\mathrm{SO}_{3}^{\prime}} .
$$

Dieses zweite Ion verschwindet schon bei sehr geringer Wasserstoffionenkonzentration und ist deshalb versuchsweise bei der Titration von Phosphorsäure verwendet worden. Hier tritt nach Bildung von primärem Salz, also z. B. $\mathrm{NaH}_{2} \mathrm{PO}_{4}$ Umsehlag von gelb zu rot ein, mithin wie auf gleicher Stufe bei Anwendung von Methylorange der Umschlag von rot zu gelb. Während aber hier Anwendung eines zweiten Indikators - Phenolphtaleïn - erforderlich ist, um die Überführung in Mononatriumphosphat anzuzeigen, tritt bei Verwendung von Alizarinsulfosäure auf dieser Reaktionsstufe der zweite Umschlag, nämlich von rot in violett ein. Der Umschlag entspricht aber ebensowenig und sogar noch weniger wie beim Phenolphtalein genau der Bildung des Dinatriumphosphates, weil dieses merklich hydrolytisch gespalten ist; der Umschlag erfolgt vorzeitig.

Eine Übergangsfarbe, in dem Sinne wie bei amphoteren Elektrolyten, also als Zwischenfarbe tritt nicht auf. Eine solche könnte aber eintreten, wenn beim Titrieren einzelner Säuren die zweite charakteristische Farbe als Endpunkt betrachtet wird; ein solcher Fall ist nicht sicher bekannt. Man könnte an die Erscheinungen beim Lackmoid und dem wahrscheinlich diesem Stoffe anzuschliefsenden Lackmus denken. Denn diese Stoffe sind mehrwertige Säuren, sodafs die Bedingungen gegeben wären.

In saurer Lösung ist die Farbe des nichtdissoziierten Molekuls, beim Lackmoid also Rot, vorhanden, bei der Neutralisation erfolgt scharfer Umschlag zu Blau. Bei Gegenwart von Kohlensäure tritt ein violetter Farbton auf, aber nicht nach dem blauen, sondern vor ihm. Es handelt sich also nicht um dieselben Erscheinungen wie 
bei der Alizarinsulfosäure, sondern der violette Farbton mufs als Mischfarbe betrachtet werden. In der Nähe des Neutralisationspunktes ist nur noch freie Kohlensäure vorhanden, d. h. sehr geringe Wasserstoffkonzentration, die schon eine teilweise Dissoziation des Lackmoids erlaubt. Umgekehrt wird eine alkaliblaue Lackmoidlösung durch eingeleitete Kohlensäure violett. Es walten also ähnliche Verhältnisse ob, wie beim Phenolphtaleyn, nur ist dieses eine schwächere Säure und die Kohlensäure kann bei genügender Konzentration völlige Entfärbung bewirken.

Nach allem gehört das Lackmoid und wahrscheinlich auch Lackmus in Gruppe A 1 und ist vor dem Phenolphtalein einzuordnen.

In die Gruppe A 1 gehört unzweifelhaft auch das Curcumin W, da es zwar verschiedene Anionen liefert, deren erstes aber mit dem nichtdissoziierten Molekul gleich, also nicht charakteristisch gefärbt ist. Gleiches gilt für das Tropäolin 000 (Azobenzol- $\alpha$-naphtolsulfosäure), wo auch ein Umschlag erst bei Bildung des Phenolions eintritt. Threr Stärke nach sind Curcumin W und Tropäolin 000 wahrscheinlich auch vor Phenolphtalein einzuordnen.

Von den durch GLASER geordneten Indikatoren sind in meine Einteilung noch fünf Indikatoren einzureihen, das Naphtobenzein, Curcuma, Hämatoxylin und das Phenacetolin, diese von unbekannter Konstitution, und schlielslich das Galleyn. Von dem ziemlich bedeutungslosen Naphtobenzein sehe ich ganz ab, vielleicht handelt es sich um eine Säure vom Charakter des Lackmoids; ebenso bieten Curcuma und Hämatoxylin kein Interesse und sind zu wenig untersucht.

Von den beiden anderen gehört zunächst das Gallern zweifellos in die Gruppe B 2, es hat verschiedene charakteristische Anionen; der Indikator scheint sogar drei derselben zu bilden. Das erste Ion gehört einer ziemlich starken Säure an und wird durch Phosphorsäure nicht verändert, starke Säuren bilden den nichtdissoziierten roten Stoff. Das zweite Ion ist rot, das dritte violett. Es ist deshalb ähnlich der Alizarinsulfosäure zur Titration von Phosphorsäure vorgeschlagen. Der Umschlag. von gelb zu rot entspricht nämlich der Bildung des Dinatriumphosphats, der weitere soll der des Trinatriumphosphates entsprechen. Da das Gallem als das erste Anhydrid eines Tetroxykörpers erscheint, also ähnlich wie Fluorescein, so ist die Bildung dreier verschiedener Anionen nicht ausgeschlossen. Vielleicht ist der Curcumafarbstoff und das Hämatoxylin hier anzureihen. 
Auch bei dem Phenacetolin liegt mit grofser Wahrscheinlichkeit ein Indikator der Gruppe B 2 vor. Der Indikator ist in saurer Lösung goldgelb, in stark alkalischer Lösung schwachgelb. Dazwischen liegt eine rote Farbe, die durch kohlensaures Alkali oder durch wenig starkes Alkali hervorgerufen wird. Wahrscheinlich entspricht das rote Anion einer ziemlich starken Säure, so dafs mit Ammoniak titriert werden kann, bei Gegenwart von viel Ammonsalz macht sich aber doch, wie wohl bei allen Indikatoren, die Hydrolyse geltend. Durch starkes Alkali kann auch das zweite Ion hervorgerufen werden, wie es scheint aber wird das hier gebildete Indikatorsalz nicht unbeträchtlich hydrolytisch gespalten. Deshalb ist die Verwendung zur Titration von Alkali neben Karbonat nur annähernd richtig. Die alkalische Lösung ist zunächst gelb, wird rot nach Neutralisation des Alkalis und gelb nach Neutralisation des Karbonats. Wegen der Hydrolyse des zweiten Indikatorsalzes tritt aber der Umschlag von gelb zu rot zu früh ein, und man findet zu wenig Alkali, zu viel Karbonat. Eigentlich müfste dieser Umschlag von gelb zu rot zu spät eintreten, weil das hydrolysierte Alkali des Karbonats mit als freies Alkali gemessen wird.

Nachdem ich so die einzelnen Indikatoren in die Gruppen meiner Einteilung eingeordnet habe, möchte ich noch zum Schlusse die Frage erörtern, in welchen Gruppen neue Indikatoren noch erwünscht wären und welche Eigenschaften sie haben müfsten, wobei das vorstehend Ausgeführte die Unterlage abgeben wird.

In Gruppe A 1 scheinen mir weitere Indikatoren überflüssig.

Wir haben in dem Jodeosin eine genügend starke Säure, um selbst sehr schwache Basen zu messen, das p-Nitrophenol genügt für Ammon; das Phenolphtaleîn ist ein idealer Indikator für starke Basen. Umgekehrt gestattet er die Messung selbst sehr schwacher Säuren mittels starkem Alkali, z. B. der Essigsäure mit $\mathrm{K}=0.00180$, ja selbst 0 - und $\mathrm{p}$-Nitrophenol mit $\mathrm{K}=0.000043$ und $\mathrm{K}=0.000012 .{ }^{1}$ Dagegen versagt nach OSTWALD der Indikator bei m-Nitrophenol mit $K=0.0000089$, so dafs die Grenze etwa bei $K=0.00001$ liegen wird. Noch schwächere Säuren malsanalytisch zu bestimmen, wird selten ein Bedürfnis vorliegen; es muls die Hydrolyse auch dann sich jeder scharfen Bestimmung entgegenstellen. Dem Übelstand, der durch die Anwesenheit von Kohlensäure verursacht wird, läfst sich durch Wegkochen begegnen.

1 Ostwald, Journ. prakt. Chem. 32, 353. 
In der zweiten Gruppe, A 2, haben wir nur sehr wenige Indikatoren, die vermehrt werden durch gewisse Indikatoren der Gruppe B 1, soweit bei ihnen vorzugsweise das basische Ion in Frage kommt. In dieser Gruppe ist noch ein Indikator erwünscht, der möglichst schwach basisch die Titration eines Karbonats mit starker Säure ermöglicht, während der Umschlag schärfer ist, wie beim Dimethylamidobenzol.

$\mathrm{Zu}$ suchen wäre dieser Indikator unter den Amidoazonaphtolen, jedenfalls unter Amidoazokörpern.

Für Indikatoren aus Gruppe B 1 kann eine Verbesserung des Methylorange erstrebt werden, damit die Empfindlichkeit dieses Indikators gegen Kohlensäure wegfällt.

Es scheint mir aber sehr wahrscheinlich, dafs mit einer dazu nötigen Verstärkung der Säurequalität ein verstärktes Vortreten der Zwischenfarbe beim Titrieren von alkalisch zu neutral verbunden ist wie beim Kongorot und dals wir auch hier das Mögliche im Methylorange erreicht haben.

Lin Bedürfnis liegt sicher vor bei Gruppe B 2 und die Erfüllung scheint insofern nicht aussichtslos, als wohl leicht - wahrscheinlich auch unter Amidooxyazokörpern oder auch unter polyvalenten Oxyazokörpern und den Phtaleïnen - Stoffe auffindbar sein werden, die zwei charakteristische Anionen von solchen Verschiedenheiten im Dissoziationsgrad der zugehörigen Säuren aufweisen, dafs wir mit ihrer Hilfe Säuren verschiedener Stärke neben einander titrieren können. Ein praktisches Bedürfnis könnte bei der Titration von Essigsäure und dergleichen neben Mineralsäuren vorliegen. $\mathrm{Da}$ wo ein Bedürfnis zweifellos vorliegt, bei der Bestimmung von Phosphat neben Phosphorsäure, andererseits bei der Bestimmung von. Karbonat neben freiem Alkali, genügen die vorhandenen Indikatoren, wie wir oben sahen, nicht. Eine Verbesserung ist möglich. Leider müssen wir aber hier von vornherein damit rechnen, dafs das Ziel nie vollkommen zu erreichen ist. Denn die hydrolytische Spaltung des Karbonats und des Dialkaliphosphats muls eine genaue Bestimmung des Neutralisationspunktes bei jedem Indikator unmöglich machen.

Leipaig, Phys.-chem. Institut, am 17. Februar 1901.

Bei der Redaktion eingegangen am 8. März 1901. 